\title{
Leaky Bloch modal evolution of wideband reflectors with zero-contrast gratings from symmetric trapezoid to triangle ridge shapes
}

\author{
Guohua Xing, ${ }^{\text {a,b }}$ Shanwen Zhang, ${ }^{\text {a,* }}$ and Robert Magnusson ${ }^{\mathrm{c}}$ \\ ${ }^{\mathrm{a}}$ Chinese Academy of Sciences, Changchun Institute of Optics and Fine Mechanics and Physics, \\ National Engineering Research Center for Diffraction Gratings Manufacturing and \\ Application, Changchun, Jilin, China \\ ${ }^{b}$ University of Chinese Academy of Sciences, Beijing, China \\ ${ }^{c}$ University of Texas at Arlington, Department of Electrical Engineering, Arlington, \\ Texas, United States
}

\begin{abstract}
We treat wideband subwavelength guided-mode resonant gratings with grating-depth dependent duty cycles. By rigorous numerical computations, we visualize Bloch modal evolution while transforming the grating profile from trapezoid to triangle. Parametric optimization is achieved using the coordinate transformation method of Chandezon. With the increase of the profile base angle, the higher mixed resonant modes $\mathrm{TM}_{1,1 \& 2}$ attract and combine and interact with the modes at both sides, forming wide reflection bands. This modal combination and interaction are the key determinant of broadband reflectivity spectral location and width. The optimized structure exhibits $99 \%$ reflectivity across a $613-\mathrm{nm}$ spectral range, spanning a 1438- to 2051-nm wavelength range with a fractional bandwidth of $\sim 35 \%$. It is shown that gratings with trapezoidal profiles possess a good tolerance to groove depth variation, thus being easier to fabricate with a diamond-tip than triangular profile-based gratings. () The Authors. Published by SPIE under a Creative Commons Attribution 4.0 Unported License. Distribution or reproduction of this work in whole or in part requires full attribution of the original publication, including its DOI. [DOI: 10.1117/1.OE .59.12.127102]
\end{abstract}

Keywords: subwavelength grating; guided-mode resonance; leaky Bloch mode.

Paper 20201003 received Aug. 21, 2020; accepted for publication Nov. 12, 2020; published online Dec. 2, 2020.

\section{Introduction}

When the phase-matching condition is satisfied between diffracted waves and leaky Bloch modes, guided-mode resonance (GMR) effects are excited on waveguide grating structures with subwavelength periods. ${ }^{1-5}$ The high reflectance characteristic produced by GMR effects can be used in frequency selection devices, biochemical sensors, broadband lossless mirrors, polarization control elements, tunable devices, and other applications. Wideband GMR reflectors with high reflectivity and polarization independence within the wideband spectrum have a higher reflectivity than many metal reflectors and simpler architecture than multilayer dielectric reflectors. Applications such as couplers, detectors, and lasers are of interest. ${ }^{6-10}$

Based on the effect of guided-mode resonance, researchers have designed a variety of grating structures, including high-contrast gratings $(\mathrm{HCG})^{11}$ and zero-contrast gratings (ZCG). ${ }^{12}$ Moreover, many gratings based on the two structures with different periods and material characteristics have been proposed in Refs. 2 and 13-15. For example, in TM polarization, the twopart periodic grating structures have been shown to exhibit $\sim 500$-nm bandwidth in Ref. 13, and the four-part periodic grating exhibits $\sim 600 \mathrm{~nm}$ in Ref. 2 . Because of the wide flat band possible with fractional bandwidth, $\Delta \lambda / \lambda_{\text {center }}$ up to $\sim 40 \%,{ }^{12}$ and ease of fabrication using mature lithography and dry etching technologies, the grating groove in these structures is mainly rectangular with the same duty cycle from the top to the bottom of the groove. ${ }^{11-17}$ In recent years, the impact of tapered sidewall profiles on the performance of GMR gratings has been studied, showing that

*Address all correspondence to Shanwen Zhang, zhshwen007@163.com 
this tapering will reduce the bandwidth and reflectivity for both ZCG and HCG. ${ }^{18,19}$ These gratings with tapered profiles have a gap between adjacent slots, which is different from the trapezoidal structures proposed here. The GMR grating with symmetrical triangle grooves is formerly presented in Ref. 20, and the optimized structures present a flat band of $\Delta \lambda / \lambda_{\text {center }} \approx$ $33.3 \%$ with $R_{0}>99 \%$ in the 1432 - to $1999-\mathrm{nm}$ wavelength range. However, the reason why the grating with triangle grooves exhibits high reflectivity in a wide spectrum range has not been specifically determined.

In this paper, to clarify the reason for the formation of wide bandwidth in triangular/ trapezoidal resonant devices, we compute modal evolution under profile variation from the symmetrical triangle to trapezoid when the thickness of grating groove changes while holding the base angle constant. We find that there are multiple higher mixed modes $\mathrm{TM}_{1,1 \& 2}$ in the transmission spectrum, which is mainly caused by the distortion of the transmission dip line in the wideband, and the convergence of these modes is the main factor for the formation of wideband. Subsequently, we demonstrate that the structures with trapezoid profiles provide a wideband reflectivity of $613 \mathrm{~nm}$ when the base angle is close to $62.8^{\circ}$. The GMR gratings with trapezoidal profiles not only have wider spectral bands than gratings with triangular profiles but are more likely to fabricate by grating ruling engines with diamond tools due to shallower groove depth and better tolerance range. ${ }^{21-23}$ Thus, these results provide an alternative structure for wideband GMR reflectors amenable to classic fabrication methods.

\section{Resonant Grating Structures}

The structures of GMR grating with triangular and trapezoidal profiles are shown in Figs. 1(a) and 1(b), respectively. Both of the structures include a homogenous layer of the same material as the grating layer with $n=3.48$ on a glass substrate with $n_{s}=1.48$. We take the materials used to be dispersionless, which is an excellent, widely-used approximation for these media and the spectral bands treated. Moreover, we assume the materials to be amorphous. The device parameters contain a grating depth $d_{g}$, homogenous-layer depth $d_{h}$, period $\Lambda$, base angle $\alpha$, and duty cycle $F$. Differing from rectangular gratings, the two types of GMR gratings have a variable duty cycle, which gradually changes with groove depth from 0 , or some other value to 1 . From the view of effective medium theory (EMT), the subwavelength grating layer can be taken to be equivalent to a multilayer structure with gradient distribution of equivalent refractive index $n_{\text {eff }}$ along the $y$ direction. The effective refractive index for each layer of triangular profiles gradually changes from $n_{c}$ to $n$ due to the varying duty cycle, which is different from rectangular grooves with a fixed index but similar to trapezoidal profiles, as shown in Fig. 1. The modal distribution in the reflection diagram in Fig. 2 can be observed clearly by decreasing the refractive index modulation. The modal distribution of gratings with triangular and trapezoidal profiles in Fig. 2(a) is different from that of gratings with rectangular profiles in Fig. 2(b), meaning that

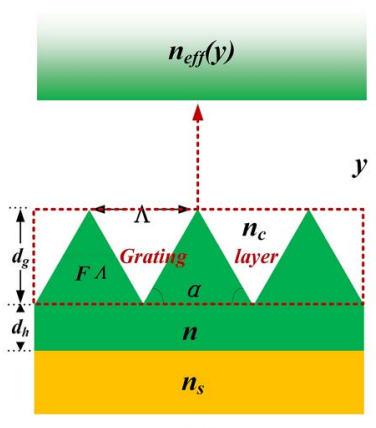

(a)

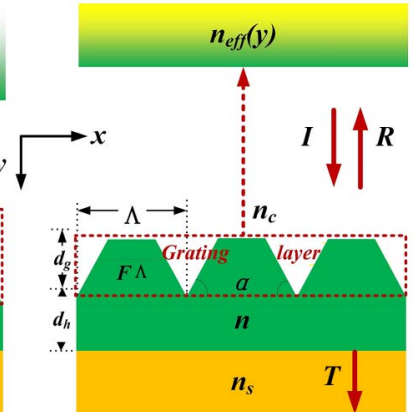

(b)

Fig. 1 Basic structures and schematic EMT index distribution of a broadband GMR reflector with (a) equilateral triangle and (b) trapezoidal profiles. The structures are made of Si with $n=3.48$ on a glass substrate with $n_{s}=1.48$, and the cover index is $n_{c}=1$ for normal incidence in the air. We represent the incident plane wave as $I, R$ as reflectance, and $T$ as transmittance. 


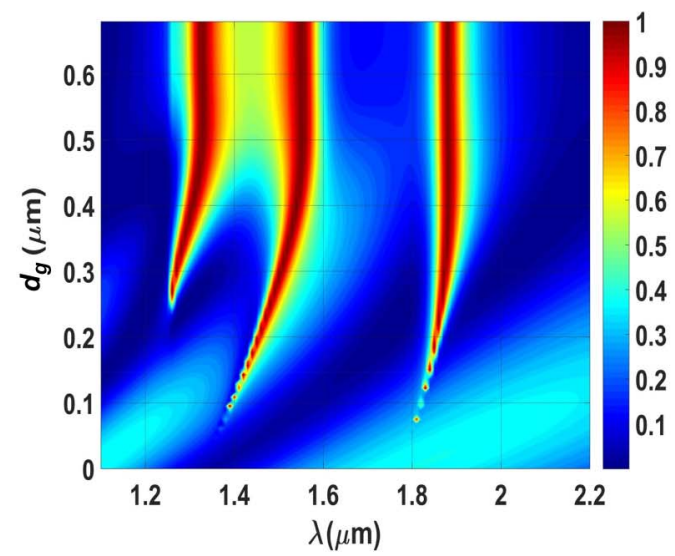

(a)

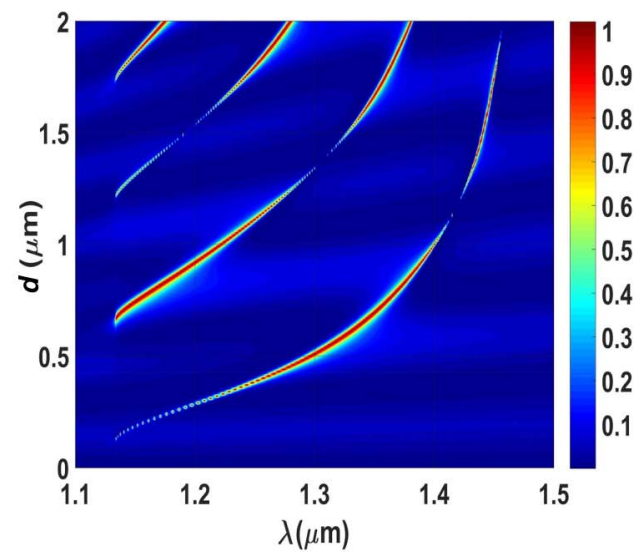

(b)

Fig. 2 (a) Reflectance map $R_{0}\left(\lambda, d_{g}\right)$ drawn versus wavelength and $d_{g}$ of the gratings with triangular and trapezoidal profiles for $\Lambda=850 \mathrm{~nm}, n_{c}=1, n=2.5, n_{s}=1.48, \alpha=58^{\circ}$, and $d_{h}=540 \mathrm{~nm}$. (b) Reflectance map $R_{0}(\lambda, d)$ of the grating with rectangle profiles for $\Lambda=766 \mathrm{~nm}, F=0.7264, n_{H}=2$, and $n_{L}=1.3417$ in Ref. 5.

the former slope more severely than the latter. The reason for the vertical mode loci in Fig. 2(a) is the dominance of the sublayer in the case of the triangular structure.

\section{Theoretical Analysis}

The optimization for these parameters of the GMR devices is achieved using the software DELTA based on the C-method. ${ }^{24,25}$ DELTA $^{26}$ was developed by Lifeng Li at Tsinghua University, and the software is used to simulate the surface relief diffraction gratings with an arbitrary refractive index. In this paper, we assume that the polarization of the incident plane wave is TM, that is, the electric-field vector is in the $x y$ plane.

\subsection{Distribution of Guided-Mode Resonances for Gratings with Equilateral Triangle Profiles}

In Ref. 20, a wideband GMR grating with symmetrical triangular grooves is proposed. The effect of the base angle and the depth of the homogeneous layer on a zero-order reflection spectrum are discussed. It is demonstrated that the bandwidth of $567 \mathrm{~nm}$ can be obtained when $n=3.48$, $\alpha=58^{\circ}$, and $d_{h}=540 \mathrm{~nm}$. However, the reason for the high reflectivity broadband was not determined. Figure 3(a) shows the reflectance map $R_{0}\left(\lambda, d_{g}\right)$ plotted against wavelength and the depth of grating with $\alpha=58^{\circ}$. The depth of the grating for the symmetrical triangular groove is about $0.68 \mu \mathrm{m}$ when $\alpha=58^{\circ}$. As can be seen from the figure, when the depth of grating is between 0.54 and $0.68 \mu \mathrm{m}$, a depth that corresponds to the black dotted box shown, the reflector has a wider bandwidth and a good tolerance range for groove depth. The transmission spectrum $T_{0}\left(\lambda, d_{g}\right)$ on a logarithmic scale drawn against wavelength and depth of grating is shown in Fig. 3(b). It can be observed that there are five resonant modes in the wideband range, which attract and interact with each other to form a broad band at $d_{g}=0.68 \mu \mathrm{m}$. This provides a reasonable explanation for the formation of the broadband of reflectors with symmetrical triangular grooves.

\subsection{Evolution Characteristics of Guided-Mode Resonant Gratings with Profiles from a Symmetric Trapezoid to a Triangle}

For the GMR grating with a symmetrical triangular groove, its base angle $\alpha$, the thickness of grating $d_{g}$, and period $\Lambda$ satisfy $d_{g}=\Lambda \tan \alpha / 2$. When $d_{g}<\Lambda \tan \alpha / 2$, the profile of the grating evolves into a trapezoid. Varying the parameters of the structures such as base angle and 


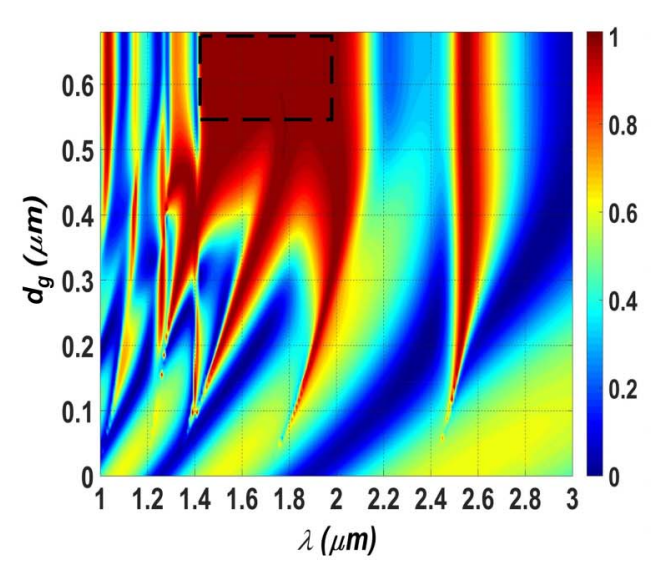

(a)

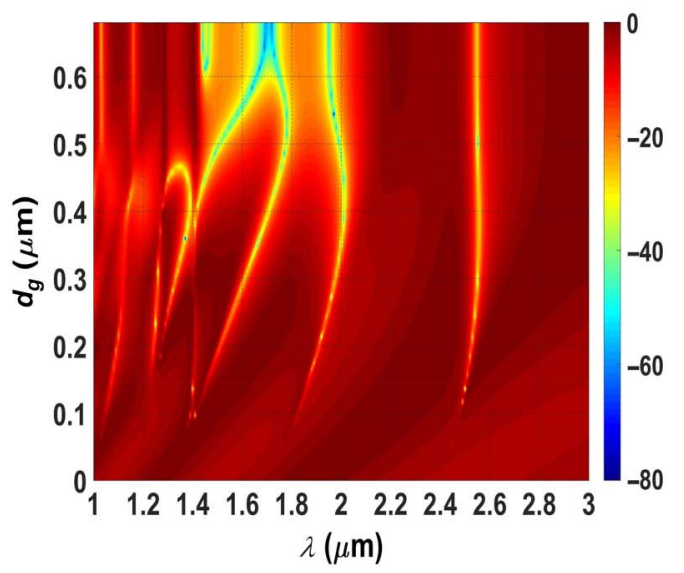

(b)

Fig. 3 (a) Reflectance map $R_{0}\left(\lambda, d_{g}\right)$ and (b) transmittance map $T_{0}\left(\lambda, d_{g}\right)$ on a logarithmic scale drawn versus wavelength and groove depth of triangle gratings. Base angles $\alpha=58^{\circ}$, $\Lambda=850 \mathrm{~nm}, n_{c}=1, n=3.48, n_{s}=1.48$, and homogenous-layer thickness $d_{h}=540 \mathrm{~nm}$, the same as the parameters in paper. ${ }^{20}$

thickness of grating, the number, and location of resonant modes will change, so a different reflection bandwidth will be observed in the reflection spectrum.

Transmission contour maps $T_{0}\left(\lambda, \mathrm{d}_{g}\right)$ on a logarithmic scale are illustrated versus wavelength and thickness for gratings with a fixed sublayer thickness and different base angles corresponding to Figs. 4(a)-4(f). The grating thickness varies from zero to $\Lambda \tan \alpha / 2$ in each case. In every map, the yellow lines with low transmission represent the distribution of resonant leaky Bloch modes, and the red region corresponds to high transmission. The fundamental mode is distributed at long wavelengths and higher modes shift to long wavelengths as the base angle $\alpha$ increases. Because the resonant modes produced at different wavelengths move at different speeds, they will meet and coincide. We denote the resonant modes by $\mathrm{TM}_{m, n}$, where $m$ represents the evanescent diffraction order and $n$ represents the order of the guided modes excited in waveguided. When $\alpha=45^{\circ}$ in Fig. 4(a), the dominant modes in the transmission spectrum are $\mathrm{TM}_{1,0}, \mathrm{TM}_{1,1}$, and $\mathrm{TM}_{1,1 \& 2}$. With the increase of the base angles, the resonant position of the fundamental modes is unchanged, whereas the position of the higher modes is dependent on the depths of the grating layer. With the increase of the grating depth, more types of modes appear and move close to each other, as shown in Figs. 4(b) and 4(c). Further variations are observed in Fig. 4(d) with $\alpha=58^{\circ}$, the two resonant modes $\mathrm{TM}_{1,1 \& 2}$ meet and combine at $d_{g}=0.68 \mu \mathrm{m}$, resulting in a strong resonant effect, which enhances the local reflection. Meanwhile, it interacts with the resonant modes on both sides and forms a broadband reflection spectrum. Therefore, the GMR grating with a triangular profile has a wide bandwidth when the base angle is $58^{\circ}$, which is consistent with results in Ref. 20. After the two modes $\left(\mathrm{TM}_{1,1<2}\right)$ meet and coincide, a single resonant peak is formed at the wavelength. Moreover, with the increase of the base angles, the full width at half maximum of the resonant peak becomes wider and the resonant intensity weakens until the resonance disappears. The resonant modes distributed in the smaller grating depth will repeat this process, which explains the drop in the position of the mode $\mathrm{TM}_{1,1 \& 2}$ in Fig. 4(e). As shown in Fig. 4(f) with $\alpha=62.8^{\circ}$, the mode $\mathrm{TM}_{1,1 \& 2}$ is mixed with the mode on the left side and recombined with an S-shaped distribution. Following the same rule as above, $\mathrm{TM}_{1,1 \& 2}$ interacts with the modes on both sides, which yields a wideband reflectivity at $d_{g}=0.55 \mu \mathrm{m}$. Therefore, the wideband reflectivity can be attributed to the combination and interaction between adjacent resonant modes, and resonant gratings with trapezoidal profiles exhibit wider bandwidth than gratings with triangular profiles. From Figs. 4(g) and 4(h) with $\alpha=64^{\circ}$ and $\alpha=70^{\circ}$, we find that the S-shaped mixed mode will be straightened and the adjacent resonant modes will separate and move away with the increase of base angles, resulting in a narrowing of the reflectivity bandwidth. It is worth noting that in Fig. 4, the grating shape is generally a trapezoid with the triangular case appearing for thickness at the top of each panel. 


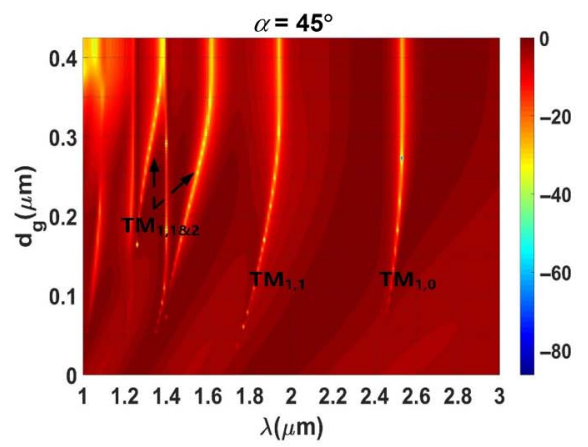

(a)

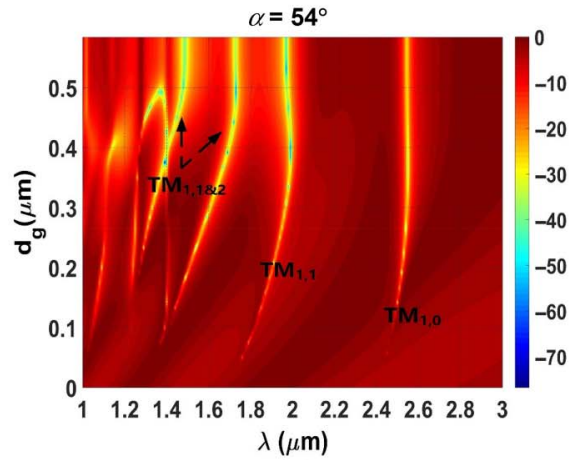

(c)

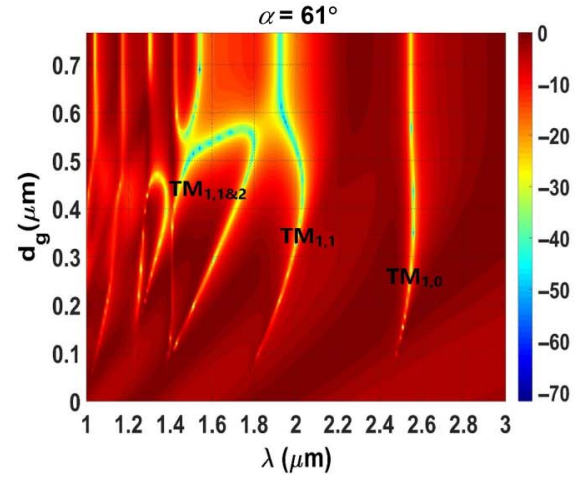

(e)

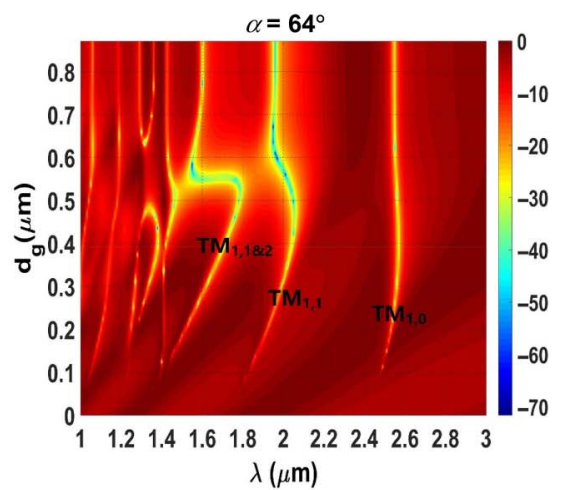

(g)

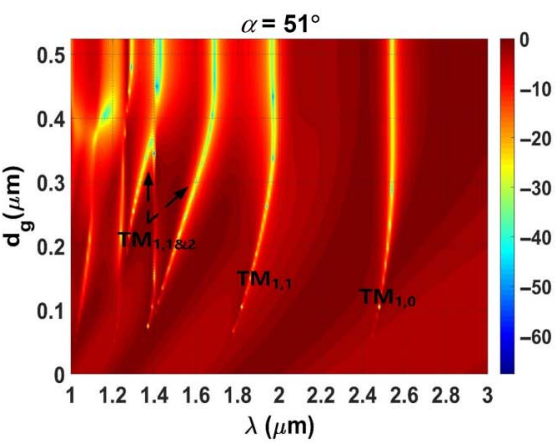

(b)

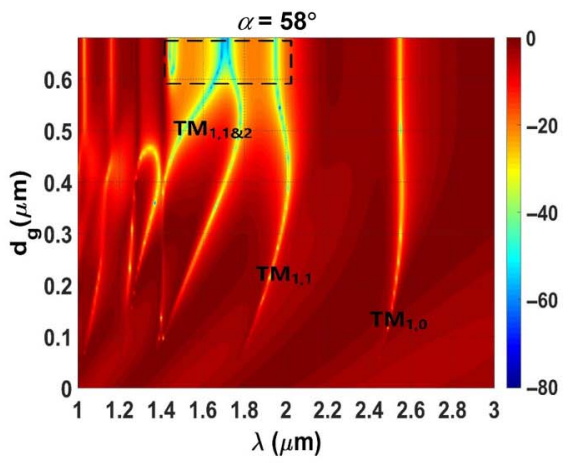

(d)

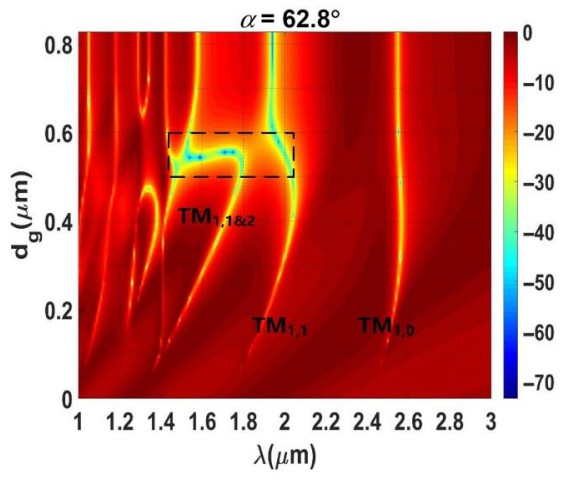

(f)

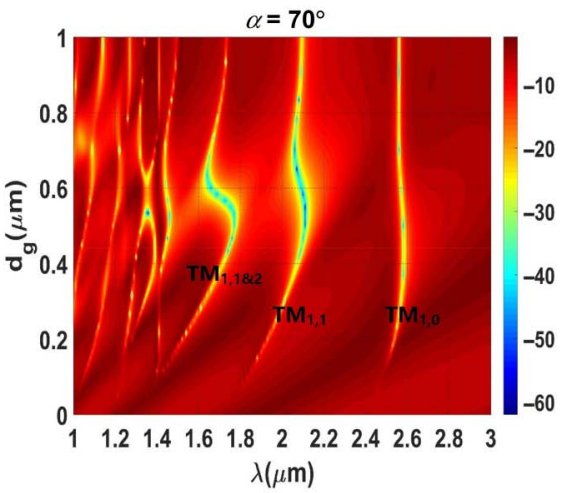

(h)

Fig. 4 Transmittance map $T_{0}\left(\lambda, d_{g}\right)$ on a logarithmic scale drawn versus wavelength and thickness of grating with triangular and trapezoidal profiles with a fixed period $\Lambda=850 \mathrm{~nm}$ and sublayer thickness $d_{h}=540 \mathrm{~nm}$. Eight different base angles $\alpha$ are considered to establish the mode dynamics and attendant reflectance spectra. (a) $\alpha=45^{\circ}$; (b) $\alpha=51^{\circ}$; (c) $\alpha=54^{\circ}$; (d) $\alpha=58^{\circ}$; (e) $\alpha=61^{\circ}$; (f) $\alpha=62.8^{\circ}$; (g) $\alpha=64^{\circ}$; (h) $\alpha=70^{\circ}$. 
The resonance structure architecture studied here has a very complex design. There is not a simple theoretical model or analytical formulation available that would bring improved transparency to the details of the physical processes at work. The multiple modes supported exist between a flat surface at the lower side and a corrugated surface at the top side. The guiding conditions differ dramatically from those of a slab waveguide or a grating between parallel planes that is the usual configuration. Nevertheless, apart from the complex mode distribution details, the lateral Bloch modes radiate across a bandwidth such that if the reradiated waves are in phase, wideband forms. This has been shown by Lalanne et al. ${ }^{3}$ in a far simpler case. The principles divulged therein are still at work in this complex case.

\subsection{Effect of the Homogeneous Thickness on Broadband Reflection Spectrum of the GMR Gratings with the Symmetric Trapezoid and Triangle Ridge Shape}

By considering the variation of the distribution of the resonant mode with the thickness of match homogeneous layer, we further clarify the reason for the formation of the wideband reflection spectrum of the GMR grating with triangular and trapezoidal grooves. Figure 5 shows the transmission contour maps versus wavelength and homogeneous depth of the GMR gratings with a triangular and trapezoidal profile on the logarithmic scale, respectively. It is obvious that with the increase of the homogeneous depth, more modes will appear and there are mainly three modes $\mathrm{TM}_{1,0}, \mathrm{TM}_{1,1}$, and $\mathrm{TM}_{1,1 \& 2}$ in the covered range, which is consistent with what we mentioned in the previous section. In the contour plot of the triangular profile, the grating is shown in Fig. 5(a), the bending of the resonant modes distribution curve occurring around a wavelength of $1700 \mathrm{~nm}$, and the homogeneous depths of $540 \mathrm{~nm}$ provides the broadest reflection band of 1432 to $1999 \mathrm{~nm}$. As shown in the transmission contour map in Fig. 5(b), the mode distribution of the trapezoidal groove grating is the same as that of the triangular groove grating, which looks like those of a GMR filter outside the broadband while being distortion within the broadband. Due to the distortion, the transmission dip line has an S-shaped distribution, which means that multiple guided-mode resonances will be excited around a homogeneous depth of $540 \mathrm{~nm}$, leading to a very broad reflection band. The distortion of the transmission dip line is caused by the strong influence of grating diffraction phase anomaly on the phase-matching condition of guided-mode resonance. ${ }^{27}$ In addition, the distribution of the mode of the trapezoidal profile grating in the wideband range is flatter than that of the triangular profile, which broadens its reflection spectrum. The distortion of the resonant mode in the wideband also explains the existence of multiple mixed-mode $\mathrm{TM}_{1,1 \& 2}$ in the transmission spectrum of Fig. 4, and the formation of the wideband is the process of the convergence of these mixed modes.

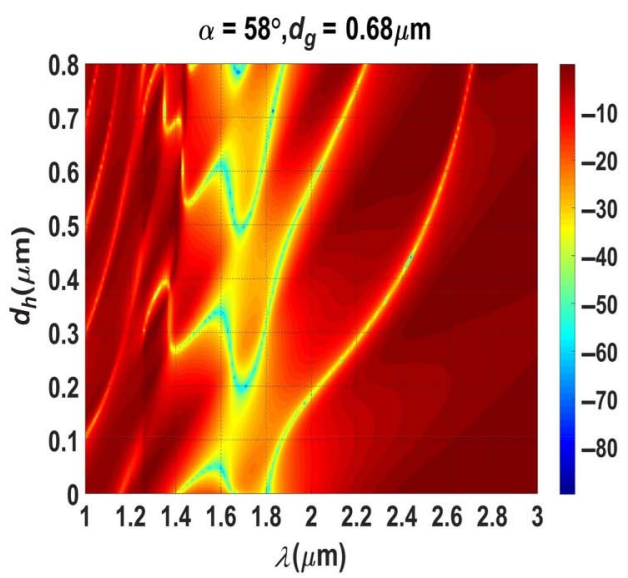

(a)

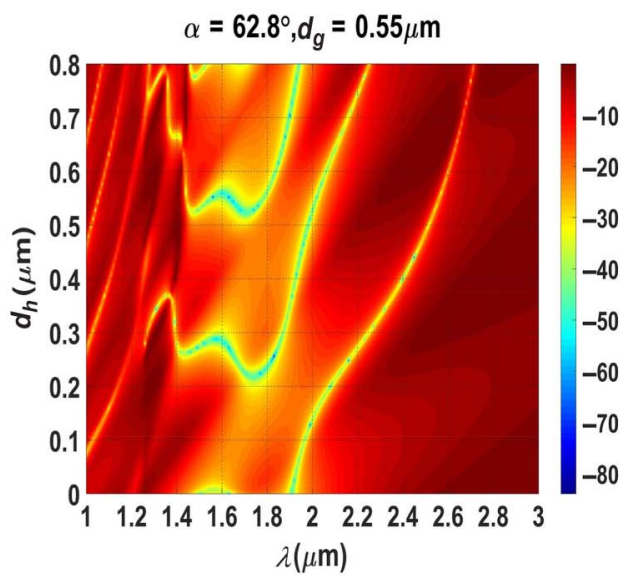

(b)

Fig. 5 Transmittance map $\mathrm{T}_{0}\left(\lambda, d_{g}\right)$ on a logarithmic scale drawn versus wavelength and thickness of homogeneous layer with (a) triangular and (b) trapezoidal profiles with a fixed period $\Lambda=850 \mathrm{~nm}$. 


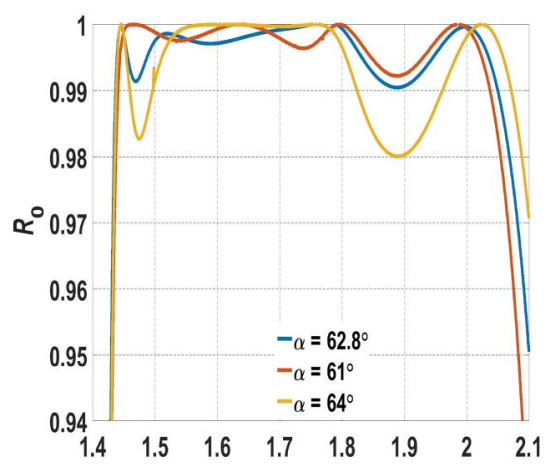

(a)

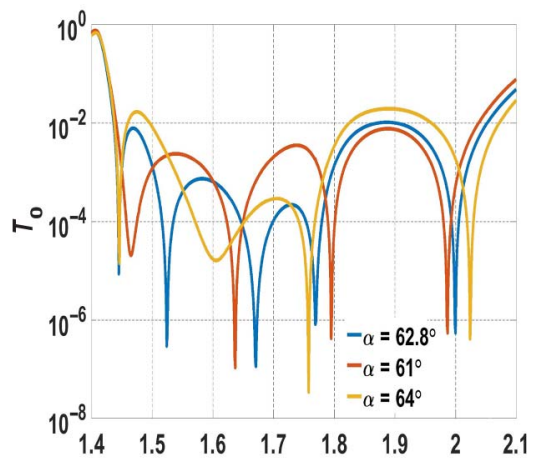

(b)

Fig. 6 Reflectance (a) linear and transmittance (b) logarithmic spectral comparison of structure with trapezoidal profile for TM polarization when $\Lambda=850 \mathrm{~nm}, n_{c}=1, n=3.48, n_{s}=1.48$, and $\alpha=62.8^{\circ} / 61^{\circ} / 64^{\circ}$.

\section{Results}

In Fig. 6, we present the maximal broadband GMR grating found in this study and its tolerance to the base angle. It has symmetrical trapezoidal grooves, and the key parameters of the maximal broadband devices for TM polarization are $\Lambda=850 \mathrm{~nm}, \alpha=62.8^{\circ}, d_{g}=550 \mathrm{~nm}$, and $d_{h}=540 \mathrm{~nm}$. The reflectance spectrum on a linear scale and the associated transmittance spectrum on a logarithmic scale are shown in Figs. 6(a) and 6(b), separately. As depicted in the blue curve in Fig. 6(a), the reflection bandwidth for $R_{0}>99 \%$ is 613-nm spanning the range of 1438 to $2051 \mathrm{~nm}$ with $\Delta \lambda / \lambda_{\text {center }}=35.1 \%$. Within the same wavelength range, there are five transmittance dips as depicted on a logarithmic scale, corresponding to the blue curve in Fig. 6(b), each of which represents a guided-mode resonance. Therefore, we can conclude that the low transmittance connected by the five resonance wavelengths form a wide flat band, which is the key to the wideband effect of this GMR reflector. In Figs. 6(a) and 6(b), the red and yellow curves correspond to $\alpha=61^{\circ}$ and $\alpha=64^{\circ}$, respectively. It is obvious that there are four resonance modes at $\alpha=61^{\circ}$, and they interact with each other to maintain the high reflectivity $(R \geq 99 \%)$ of the structure from 1441 to $2037 \mathrm{~nm}$. Similarly, there are four resonant modes at $\alpha=64^{\circ}$, but the resonance intensity of the two resonant modes in the short wavelength direction weakens, resulting in a small decrease in the local reflectivity around the $1425 \mathrm{~nm}$. On the other hand, the distance between the two resonant modes in the long-wavelength direction is large and the interaction between them is weakened, resulting in a small decrease in the local reflectivity around $1890 \mathrm{~nm}$. Overall, for these example angular deviations, the reflectance varies $<2 \%$ across the band of interest as seen in Fig. 6(a).

Figures 7(a)-7(f) show the pattern of refractive index and magnetic field inside the grating and surrounding media at the five resonant wavelengths, which helps to further clarify the distribution of resonant modes and the formation of wideband. At the short-wavelength $\lambda=1.445 \mu \mathrm{m}$, which is less than the cutoff wavelength of the second-order diffracted wave $\left(\lambda_{2, \text { cutoff }}=\Lambda n_{\text {eff }} / 2\right)$, there are not only the guided modes excited by the first-order diffracted wave $\mathrm{TM}_{1, n}$ but also excited by the second-order diffracted wave $\mathrm{TM}_{2, n}$, so the distribution of the modes is more complex. It is distributed at the junction of the grating layer and the matched homogeneous layer and inside the homogeneous layer. When $\lambda=1.524 \mu \mathrm{m}$ in Fig. 7(c), the field is pushed into the grating layer. In Fig. 7(d) of wavelength $\lambda=1.671 \mu \mathrm{m}$, the magnetic field periodically distributes in the homogenous layer and the grating layer and shows the characteristics of mixed-mode $\mathrm{TM}_{1,1 \& 2}$. We show that the emergence of mixed-mode $\mathrm{TM}_{1,1 \& 2}$ is mainly caused by the difference in the thickness of the waveguide layer. The field distribution in Fig. 7(e) is the same as that in Fig. 7(d), showing the characteristics of $\mathrm{TM}_{1,1 \& 2}$, except that the field amplitude in the grating layer is weakened. At the long-wavelength $\lambda=2.000 \mu \mathrm{m}$ in Fig. 7(f), the field is distributed in the homogenous layer and the bottom of the grating layer, and this distribution is periodic along the $\mathrm{x}$ and $\mathrm{z}$ directions, showing the characteristics of $\mathrm{TM}_{1,1}$. 


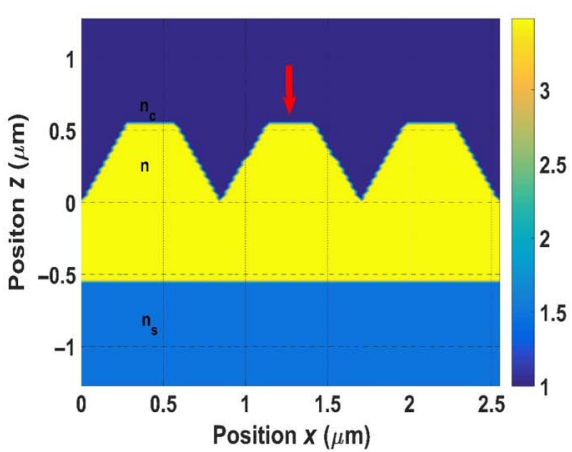

(a)

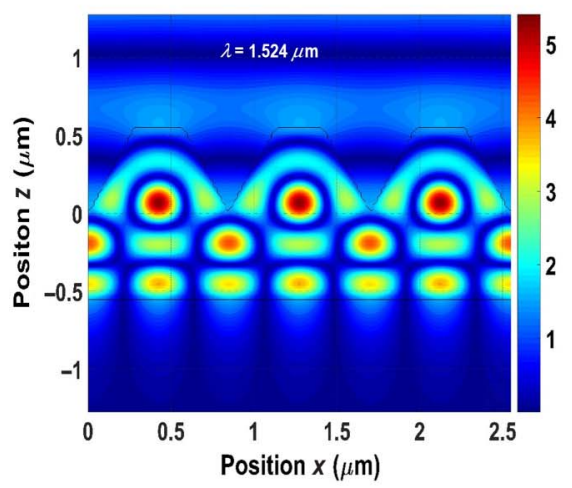

(c)

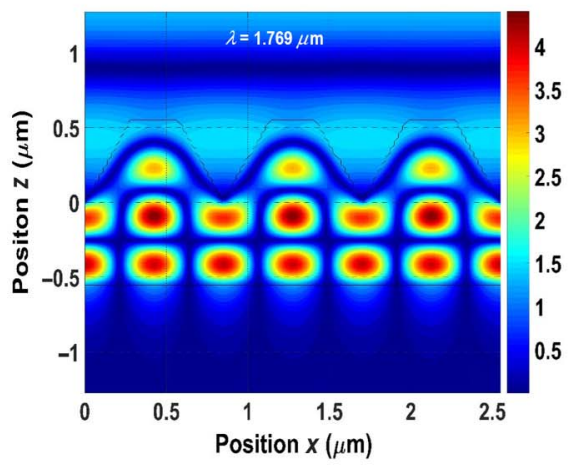

(e)

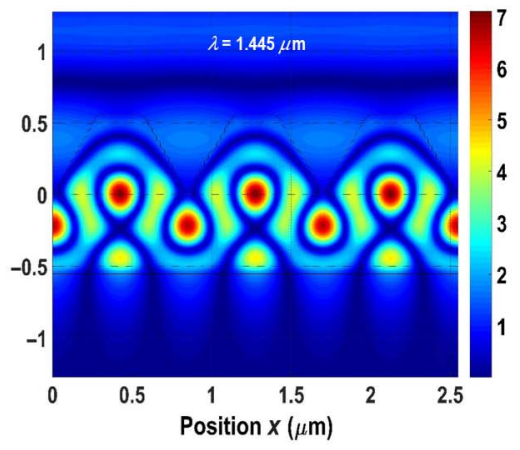

(b)

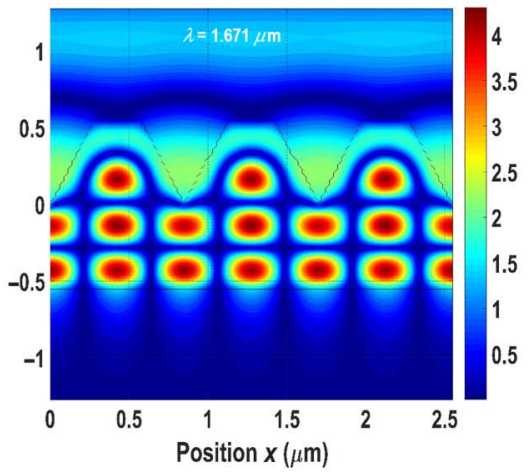

(d)

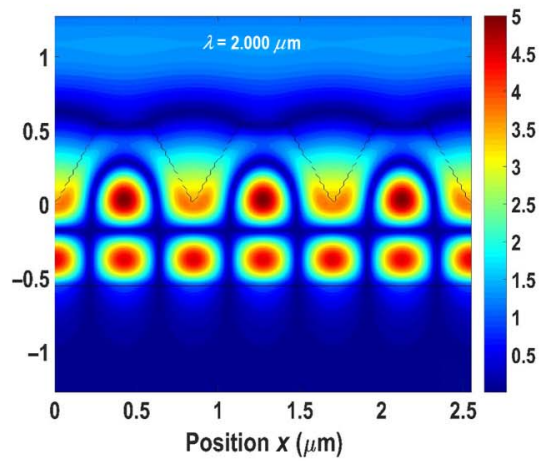

(f)

Fig. 7 (a) The distribution of refractive index and amplitude of the internal magnetic field in the GMR grating with trapezoidal profiles and surrounding media at the resonance wavelengths for $\Lambda=850 \mathrm{~nm}, \alpha=62.8^{\circ}, d_{h}=540 \mathrm{~nm}$ and $d_{g}=550 \mathrm{~nm}$. (b) $\lambda=1.445 \mu \mathrm{m}$; (c) $\lambda=1.524 \mu \mathrm{m}$; (d) $\lambda=1.671 \mu \mathrm{m}$; (e) $\lambda=1.769 \mu \mathrm{m}$; (f) $\lambda=2.000 \mu \mathrm{m}$.

Resonant wideband reflectors, in general, can be compared to Bragg reflectors. Distributed Bragg reflectors (DBRs), which consist of multiple layers of materials with alternating high and low refractive index, often 10 to 100 layers, have been widely used as high reflectivity mirrors in surface-emitting lasers, for example. The main practical difference between the resonant reflectors and DBRs lies in the layer count. Other attributes have been compared as discussed in detail in the literature. ${ }^{28,29}$

\section{Conclusion}

In this paper, broadband GMR gratings with a depth-dependent duty cycle, including symmetrical triangular and trapezoidal profiles, are designed and their modal evolution simulated under a variable base angle. A grating with a trapezoidal profile is found to provide a 613-nm spectral width for $99 \%$ reflectance, a $\sim 46-\mathrm{nm}$ enhancement relative to triangular profiles. ${ }^{20}$ From the 
transmission spectrum, we observe the combination of mixed modes $\mathrm{TM}_{1,1 \& 2}$ and the interaction with other modes, with the increase of the base angle, explaining why quasi-equilateral triangular and trapezoidal grating profiles achieve broadband features at optimized parametric values. As the base angle continues to increase, the mixed-mode with S-shaped distribution will gradually straighten and reduce the bandwidth. For the complex device architectures treated here, there exist no simple analytical formulations; hence, rigorous numerical methods are essential for the discovery of functional devices with superior performance. With a shallower groove depth and better tolerance range, gratings with trapezoidal profiles are easier to be fabricated by grating ruling engines that would be imbued with a high-resolution diamond-tipped scribes. Thus, the conclusions of this study have practical ramifications.

\section{Acknowledgments}

The authors acknowledge supports from the Fudan University-CIOMP Joint Fund (Grant No. Y9S133H190), and the International cooperation project (Grant No. Y8E43WH). The authors declare no conflicts of interest.

\section{References}

1. D. Rosenblatt, A. Sharon, and A. A. Friesem, "Resonant grating waveguide structures," IEEE J. Quantum Electron. 33(11), 2038-2059 (1997).

2. Y. Ding and R. Magnusson, "Resonant leaky-mode spectral-band engineering and device applications," Opt. Express 12(23), 5661-5674 (2004).

3. P. Lalanne, J. P. Hugonin, and P. Chavel, "Optical properties of deep lamellar gratings: a coupled Bloch-mode insight," J. Lightwave Technol. 24(6), 2442-2449 (2006).

4. Y. H. Ko and R. Magnusson, "Wideband dielectric metamaterial reflectors: Mie scattering or leaky Bloch mode resonance?" Optica 5(3), 289-294 (2018).

5. R. Magnusson and M. Shokooh-Saremi, "Physical basis for wideband resonant reflectors," Opt. Express 16(5), 3456-3462 (2008).

6. S. S. Wang and R. Magnusson, "Theory and applications of guided-mode resonance filters," Appl. Opt. 32(14), 2606-2613 (1993).

7. M. C. Y. Huang, Y. Zhou, and C. J. Chang-Hasnain, "A surface-emitting laser incorporating a high index-contrast subwavelength grating," Nat. Photonics 1, 119-122 (2007).

8. P. Cheben et al., "A broad-band waveguide grating coupler with a subwavelength grating mirror," IEEE Photonics Technol. Lett. 18(1), 13-15 (2006).

9. C. C. Wang and S. D. Lin, "Resonant cavity-enhanced quantum-dot infrared photodetectors with sub-wavelength grating mirror," J. Appl. Phys. 113, 213108 (2013).

10. C. P. Sturmberg et al., "Fano resonances of dielectric gratings: symmetries and broadband filtering," Opt. Express 23(24), A1672-A1686 (2015).

11. C. F. R. Mateus et al., "Broad-band mirror $(1.12-1.62 \mu \mathrm{m})$ using a subwavelength grating," IEEE Photonics Technol. Lett. 16(7), 1676-1678 (2004).

12. R. Magnusson, "Wideband reflectors with zero-contrast gratings," Opt. Lett. 39(15), 4337-4340 (2014).

13. C. F. R. Mateus et al., "Ultrabroadband mirror using low-index cladded subwavelength grating," IEEE Photonics Technol. Lett. 16(2), 518-520 (2004).

14. A. Taghizadeh et al., "Hybrid grating reflector with high reflectivity and broad bandwidth," Opt. Express 22(18), 21175-21184 (2014).

15. M. Shokooh-Saremi and R. Magnusson, "Wideband leaky-mode resonance reflectors: influence of grating profile and sublayers," Opt. Express 16(22), 18249-18263 (2008).

16. H. Wu et al., "A multilayer-based high-performance multisubpart profile grating reflector," IEEE Photonics Technol. Lett. 22(4), 203-205 (2010).

17. H. Wu et al., "A wideband reflector realized by a subwavelength multi-subpart profile grating structure," J. Opt. 15, 035703 (2013).

18. W. Yu, M. Ye, and Y. S. Yi, "Impacts of tapered sidewall profile on subwavelength grating wideband reflectors," J. Nanophotonics 9, 093058 (2015). 
19. X. Y. Wenxi and Y. Yi, "Impacts of tapered sidewall profiles with high aspect ratio on subwavelength grating structure," IEEE Photonics Technol. Lett. 27(13), 1437-1440 (2015).

20. S. Zhang, Y. H. Ko, and R. Magnusson, "Broadband guided-mode resonant reflectors with quasi-equilateral triangle grating profiles," Opt. Express 25(23), 28451-28458 (2017).

21. X. Li et al., "300 mm ruling engine producing gratings and Echelles under interferometric control in China," Appl. Opt. 54(7), 1819-1826 (2015).

22. Jirigalantu et al., "Ruling of Echelles and gratings with a diamond tool by the torque equilibrium method," Appl. Opt. 55(28), 8082-8088 (2016).

23. S. Zhang et al., "Groove shape characteristics of Echelle gratings with high diffraction efficiency," Opt. Commun. 387, 401-404 (2017).

24. J. Chandezon, D. Maystre, and G. Raoult, "A new theoretical method for diffraction gratings and its numerical application," J. Opt. 11(4), 235-241 (1980).

25. J. Chandezon et al., "Multicoated grating: a differential formalism applicable in the entire optical region," J. Opt. Soc. Am. 72(7), 839-846 (1982).

26. L. Li et al., "Rigorous and efficient grating-analysis method made easy for optical engineers," Appl. Opt. 38(2), 304-313 (1999).

27. G. C. Park, A. Taghizadeh, and, , and I. S. Chung, "Hybrid grating reflectors: origin of ultrabroad stopband," Appl. Phys. Lett. 108(14), 141108 (2016).

28. K. J. Lee and R. Magnusson, "Single-layer resonant high reflector in TE polarization: theory and experiment," IEEE Photonics J. 3(1), 123-129 (2011).

29. M. Niraula, J. W. Yoon, and R. Magnusson, "Single-layer optical bandpass filter technology," Opt. Lett. 40(21), 5062-5065 (2015).

Guohua Xing is a PhD student at the University of the Chinese Academy of Sciences. She received her BS degree in applied physics from Yantai University in 2018. Her current research interests include GMR gratings.

Biographies for the other authors are not available. 